\title{
Matrin3: connecting gene expression with the nuclear matrix
}

\author{
Miguel B. Coelho ${ }^{1}$, Jan Attig ${ }^{2}$, Jernej Ule ${ }^{2}, \&$ Christopher WJ Smith ${ }^{1 *}$ \\ ${ }^{1}$ Department of Biochemistry, University of Cambridge, Tennis Court Road, Cambridge, CB2 1QW, UK \\ ${ }^{2}$ Department of Molecular Neuroscience, UCL Institute of Neurology, Queen Square, London, WC1N \\ 3BG, UK \\ * Corresponding author: cwjs1@cam.ac.uk
}


Abstract

As indicated by its name, Matrin3 was discovered as a component of the nuclear matrix, an insoluble fibrogranular network that structurally organizes the nucleus. Matrin3 possesses both DNA and RNA binding domains and, consistent with this, has been shown to function at a number of stages in the life-cycle of mRNAs. These numerous activities indicate that Matrin3, and indeed the nuclear matrix, do not just provide a structural framework for nuclear activities, but play direct functional roles in these activities. Here we review the structure, functions, and molecular interactions of Matrin3 and of Matrin3-related proteins, and the pathologies that can arise upon mutation of Matrin3.

\section{Introduction}

Matrin3 was first identified as a major component of the nuclear matrix ${ }^{1,2}$, which connects the nuclear membrane and the lamin class of intermediate filaments to intra-nuclear structures (see Box 1). The nuclear matrix acts as a scaffold for attachment of chromatin, both active and inactive, binding to specific regions on the chromosomes termed scaffold/matrix attachment regions (S/MARs) ${ }^{3}$. The S/MARs have been associated with various functions including regulation of transcription ${ }^{4,5}$, RNA processing ${ }^{6}$, insulation of genomic domains ${ }^{7}$ and facilitating DNA replication ${ }^{8}$. Thus, attachment of chromatin to the nuclear matrix not only spatially organizes the nucleus, but also contributes to numerous nuclear functions.

\section{Box 1. The nuclear matrix}

The nuclear matrix consists of a non-chromatin fibrogranular ribonucleoprotein network located within the nucleus but connecting with the internal surface of the nuclear membrane, interior to the nuclear lamina. Sometimes called the nuclear scaffold or skeleton, historically the nuclear matrix has been defined in two complementary ways. First, and most importantly, electron microscopy allowed direct visualization of an extensive non-chromatin branched fibrogranular ribonucleoprotein network that had not been evident in earlier analyses by light microscopy ${ }^{9}$. Biochemical approaches identified the matrix as a structure resistant to sequential extractions, including high concentrations of salt, non-ionic detergents and digestion with DNase. These treatments remove both soluble complexes such as the spliceosome as well as some insoluble complexes including chromatin. The general structure of the nuclear matrix is insensitive to DNase treatment, emphasizing the identity of the matrix as a separate entity from chromatin, albeit one that is proposed to organize 
chromatin loops via interaction at S/MARs. Conversely, the matrix is sensitive to RNase digestion, indicating that ribonucleoprotein complexes are integral to the structure, and presumably functions, of the matrix ${ }^{10}$. One of the major challenges in understanding the nuclear matrix is defining the major protein constituents that underlie the observed filamentous structure; proteomic analyses indicate a highly complex composition ${ }^{11}$, including many RNA binding proteins. The matrix-association of proteins known to be active in various nuclear processes argues for the functional importance of the matrix. Nevertheless it should be noted that the concept of a nuclear matrix is not universally accepted ${ }^{12,13}$.

Matrin3 was identified by analysis of the protein complement of the biochemically defined nuclear matrix fraction ${ }^{1}$. To characterize the key players of the nuclear matrix, Berezney and colleagues used two-dimensional polyacrylamide gels to separate and identify the major protein components of the extracted nuclear matrix from rat liver cells ${ }^{1}$. Together with the lamins $A, B$ and $C$, other abundant proteins were identified, including Matrins 3, 12, 13, D/E, F/G (Prostaglandin transporter SLC21A2), PSF/SFPQ (at the time named Matrin4) and nucleophosmin (also known as B-23), as well as several hnRNPs. Subsequently, many other proteins have been found to localize to this structure and have been identified using quantitative proteomic tools ${ }^{11}$. Taking a complementary approach Sugano and colleagues identified a $130 \mathrm{kDa}$ protein, initially named P130 after its gel migration size, which binds to AT-rich sequences found in highly repetitive matrix-associated DNA sequences, and upon protein sequence analysis revealed to be Matrin3 ${ }^{14,15}$.

Although a major component of the matrix that provides structure and organization to the nucleus, the majority of the 847 amino acids of Matrin3 comprise intrinsically disordered regions, with the exceptions of two zinc-finger domains and two tandem RNA binding domains ${ }^{16}$. The assumption that its matrix localization implies a passive structural role could not be further from the truth; Matrin3 has been associated with functions ranging from transcription regulation, pre-mRNA splicing, mRNA stability, DNA damage repair to cell proliferation (see below). The involvement of Matrin3 and other nuclear matrix proteins in these processes suggests that the nuclear matrix might have important roles not only in the spatial organization and compartmentalization, but also in the activity of the specialized machineries involved in these various nuclear functions. 


\section{Domain structure and function of Matrin3}

Four distinguishable structured domains are present in Matrin3 (Fig. 1A), two DNA binding $\mathrm{C}_{2} \mathrm{H}_{2}$ zinc-finger domains (ZF) and two RNA binding domains of the RNA recognition motif type $(\mathrm{RRM})^{16}$. In addition to these domains there are also a bipartite nuclear localization signal (NLS) and a nuclear export signal (NES) $)^{16,17}$. Most of the remaining protein sequence is predicted to be disordered, and therefore might be dedicated to mediating protein-protein interactions, as motifs for protein interactions are commonly found in disordered regions ${ }^{18}$. One such motif is a PTBP1 RRM interaction motif (PRI), a 7 amino acid motif that mediates interaction with the splicing regulator polypyrimidine tract binding protein (PTBP1) and possibly other proteins $^{19}$.

Along with Lamin B, Matrin3 is one of the few nuclear matrix proteins that do not bind to total genomic DNA from rat liver, when analysed for DNA binding by southwestern blot $^{20}$. Nevertheless, subsequent studies have shown that Matrin3 can bind to at least some DNA sequences ${ }^{14-16}$. The two zinc-finger (ZF) domains belong to the U1-like zinc finger family although in contrast to the member that gives name to the family, $\mathrm{U} 1 \mathrm{C}^{21}$, the $\mathrm{ZF}$ domains in Matrin3 mediate interaction with DNA ${ }^{16}$. The two ZF domains show some redundancy as deletion of both ZF1 and ZF2 is required to impair DNA binding, assayed by electrophoretic mobility shift assay (EMSA), and recruitment to chromatin in cells or in biochemically fractionated chromatin ${ }^{16}$. The ZF domains cannot compensate binding to RNA when the RRM domains are deleted ${ }^{16}$. The ZF domains are possibly associated with roles in chromosome positioning, transcription regulation and/or genic insulation as all these typically involve DNA binding proteins. Deletion of the ZF domains does not affect the general role of Matrin3 in alternative splicing regulation, but it remains possible that it interacts with DNA to regulate specific co-transcriptional RNA processing events ${ }^{19}$.

The two RRM domains of Matrin3 are most similar to the RRM domains of PTBP1 and hnRNPL ${ }^{16}$, with $37 \%$ sequence identity to the first of the four RRM domains of PTBP1. The AUCUU motif was determined as the optimal RNA binding site for the tandem RRMs of Matrin3 by RNA-compete array-based selection as part of a study characterizing the binding sites for 207 RNA binding proteins ${ }^{22}$. Whether this motif reflects optimal binding to one or both RRMs currently remains unclear, since the 5-mer length of this motif is consistent with interaction of a single RRM 
domain $^{23}$. There is evidence that some RNA-dependent protein interactions require the second RRM but not the first, suggesting that RRM2 might play the dominant role in RNA binding ${ }^{24}$. The AUCUU motif is similar to the UUUCU motif bound by PTBP1, which directly interacts with Matrin3 and cooperates with it in alternative splicing regulation. The two tandem RRMs of Matrin3 bind to the regulated RNA targets and are required for its role in splicing regulation and RNA stabilization ${ }^{19,} 24$. RNA map constructed by mapping Matrin3 iCLIP tags onto the Matrin3 regulated splicing events suggests that in vivo RNA binding might involve initial binding to specific sites followed by oligomerization along the $\operatorname{RNA}^{25}$ (see below).

Immunofluorescence microscopy found Matrin3 as a mainly nuclear protein in various mammalian cell lines, where it localizes throughout the nucleoplasm with the exception of the nucleolus ${ }^{26,27}$. Nuclear matrix localization of proteins like SATB1, Runx and AML-1 is mediated through a nuclear matrix targeting sequence (NMTS) distinct from the NLS ${ }^{28,29}$, but a functionally equivalent motif could not be identified in Matrin3. Although Matrin3 was originally discovered by its presence in nuclear matrix fractions, biochemical fractionation and western blotting show it is also present in soluble nucleoplasmic fractions ${ }^{16,19}$, and to some extent also in cytoplasmic fractions, albeit at lower levels than in the nucleus ${ }^{16}$. The lack of an identified NMTS in Matrin3 means that it is not yet possible to determine which of its nuclear functions depend on its matrix localization. Shuttling between the nucleus and cytoplasm is mediated by the nuclear export signal (NES) located at the N-terminus, and the bipartite nuclear localization signal (NLS) located between the second RRM and the second ZF domain ${ }^{16,17}$ (Fig.1A). Although no evidence for a cytoplasmic role has yet been described, the presence of a NES and some cytoplasmic localization suggest that such a role may exist.

\section{Molecular interactions of Matrin3}

Matrin3 can interact with multiple nuclear proteins, a high proportion of which are involved in RNA binding and/or processing (Table 1). Some of these are important for Matrin3 function, including the interactions with PTBP1, NONO (also known as p54nrb) and PSF (reviewed $\mathrm{in}^{30}$ ), but the function of other interactions remains unclear. The domains and motifs mediating most direct protein-protein 
interactions remain unclear, with one exception being the PRI-mediated interaction with PTBP $1^{19}$. In fact, some of the reported interactions might be indirectly bridged by RNA, since they were identified by techniques that do not distinguish between direct and RNA-bridged interactions, including yeast two hybrid ( $\mathrm{Y} 2 \mathrm{H})$ and immunoprecipitation (IP) without nuclease treatment (Table 1). Moreover, interaction sites for binding partners may overlap, and their competition for Matrin3 binding might be relevant for the different Matrin3 functions. Some of these interactions may require presence of Matrin3 within the nuclear matrix, which could serve as a scaffold for specific functions. The interaction between Matrin3 and LaminA ${ }^{26}$ is one such matrix associated interaction. Matrin3 binds to the tail of LaminA and immunofluorescence shows co-localization on the nuclear rim of differentiated C2C12 myotubules, although it is unclear if this is in the nuclear membrane or nuclear matrix. This interaction might be involved in the anchoring of the nuclear envelope bound lamins to the nuclear matrix, thereby ensuring structural integrity of the nucleus.

\section{Matrin3-related proteins}

Mammals possess two Matrin3-related proteins, RBM20 and ZNF638, which share its overall domain organization, including the related ZF and RRM domains (Fig. 1A). While Matrin3 and ZNF638 are widely expressed across different cell types, RBM20 expression is mostly restricted to the foetal and adult heart (Fig 1B). ZNF638 (originally known as NP220) has a pair of RRMs between the two $\mathrm{C}_{2} \mathrm{H}_{2} \mathrm{ZF}$ domains $^{31}$. Sequence identity is highest in the ZF and RRM domains, although RBM20 only has a single RRM domain. RBM20 shares $29 \%$ amino acid identity with Matrin3 over $43 \%$ of its length. Mapping of the RNA targets of RBM20 by CLIP revealed an optimal UCUU binding motif ${ }^{32}$, which is remarkably similar to the optimal binding sites for both Matrin3 (AUCUU) ${ }^{22}$, and PTBP1 (UUUCU/UCUU) ${ }^{22,33}$. This is consistent with the fact that the first RRM of PTBP1 recognises YCU motif ${ }^{34}$ and has a high degree of identity with the RRMs of Matrin3 (40\% and $35 \%$ ) and RBM20 (51\%). RBM20 and ZNF638 lack the PRI motif found in Matrin3, and so presumably do not interact with the splicing regulator PTBP1. Nevertheless, both proteins were 
found to regulate splicing ${ }^{35,36}$, and have regions enriched in RS dipeptides, a feature commonly associated with splicing factors and regulators

\section{Matrin3 function in transcriptional regulation}

A number of functions have been suggested for Matrin3 throughout the life cycle of mRNAs, from the regulation of transcription, alternative splicing, viral RNA export, mRNA stability, to the DNA damage response. The role of Matrin3 in transcriptional regulation originates from the finding that Matrin3 interacts with the regions of DNA that are present within the scaffold/matrix attachment regions (S/MARs) ${ }^{3}$. These Matrin3-bound DNA regions augmented transcription when placed upstream of a promoter in a reporter plasmid ${ }^{37}$. Matrin3 binding decreased upon methylation of this DNA region, with a corresponding decrease in transcription, thereby suggesting that Matrin3 can activate transcription ${ }^{38}$. Matrin3 might not be the only factor involved in transcriptional activation by these DNA regions, given that these regions were isolated from the nuclear matrix scaffold that contain many proteins involved in transcription ${ }^{11,37}$. Matrin3 was also found to bind to Pit1, a transcription factor involved in the activation of multiple genes, most notably the pituary hormone gene. Enhancer-bound Pit1 has to interact with the nuclear matrix to recruit the transcription factors beta-catenin and SATB1 to specific enhancer loci. The interaction of Pit1 with the nuclear matrix and Matrin3, requires beta-catenin and SATB1, and in absence of these factors Pit1 is unable to activate transcription. A disease-causing dominant negative mutation of Pit1 (R271W) disrupts the interaction with beta-catenin and SATB1, and consequent loss of association of enhancer occupied Pit-1 with the nuclear matrix. This interaction loss can be rescued by fusing Pit1 R271W to the nuclear matrix targeting region from hnRNPU/SAF-A, with the remarkable rescue of enhancer activation and recruitment of transcription coactivators such as $\mathrm{p} 300$, and consequent transcription activation ${ }^{39}$.

Transcription is thought to occur in a limited number of "transcription factories" at fixed locations in the nucleus ${ }^{40}$, which implies anchoring to a structural component that limits diffusion. Besides Matrin3, other components of the nuclear matrix impact transcription, positively and negatively. While Lamin B is associated with insulator sequences $^{41}$, matrix-associated RNA-binding binding proteins seem to aid in 
maintaining a transcriptional active environment. The nuclear matrix component hnRNPU is associated with sites of RNA pol II transcription ${ }^{42}$, and expression of a dominant negative mutant of hnRNPU destabilises the matrix in a similar manner as does RNase treatment ${ }^{43}$. Matrin3 has been found at RNA pol II initiation sites together with hnRNPU, highlighting the involvement of Matrin3 in a network of transcriptional activators ${ }^{44}$.

\section{Matrin3 function in splicing regulation}

Generation of alternative spliced mRNAs allows cells to expand their proteome ${ }^{45}$. Transcriptome-wide analysis of changes in alternative splicing upon Matrin3 depletion in HeLa cells revealed that Matrin3 regulates many alternative exons, mostly as a direct repressor but in some cases also as an activator ${ }^{19}$. While Matrin3 required its RRMs for splicing activation, no evidence of direct binding was found around the enhanced exons, suggesting that this function might be indirect. Matrin3 was identified as one of the main binding partners of the PTBP1 (Table 1), interacting with the second RRM domain of PTBP $1^{19}$. This interaction is mediated through a specific small linear motif GILGPPP found between Matrin3's first ZF and the RRMs. This sequence conforms to a consensus PRI ( $\underline{\text { TTP}} 1 \underline{\mathrm{R} R M}$ Interaction) motif, originally identified in the PTBP1 co-regulator Raver1 and shown to dock onto the dorsal surface of PTBP1 RRM2 ${ }^{46,47}$, a domain important for PTBP1 repressor activity ${ }^{48,49}$. Despite this route of identification, comparison between splicing events regulated by Matrin3 or $\mathrm{PTBP} 1^{50}$ revealed that only a subset of events are coregulated by both proteins. This indicates that Matrin3 regulates most of its targets independently of PTBP1. Nevertheless, the activity of Matrin3 as a splicing regulator

requires its PRI motif, for both PTBP1-dependent and -independent events ${ }^{19}$ (and unpublished observations). This raises the hypothesis that Matrin3 might not only interact with PTBP1, but also with other co-regulatory proteins through its PRI motif, depending on the regulated splicing event. In fact, Matrin3 can interact with multiple RNA binding proteins (see Table 1) although it is not yet known which are involved in splicing co-regulation.

The mechanism of splicing repression was examined by mapping Matrin3 binding sites with nucleotide-resolution crosslinking immunoprecipitation (iCLIP). 
Interestingly, Matrin3 interacts with broad sections of the pre-mRNA within the repressed exons and the flanking introns. In contrast to the splicing maps for many other splicing regulators ${ }^{25}$, Matrin3 binding was relatively uniform over a region encompassing 500 nucleotides flanking the repressed exons, with no specific peaks of binding. Matrin3 RNA-compete motifs were also enriched in the flanking introns, but not within the repressed exons ${ }^{19}$. This suggests a "bind and spread" model in which Matrin3 is recruited initially to the intronic high affinity binding sites, with subsequent recruitment of other Matrin3 molecules that interact and spread across the exon and flanking introns (Fig. 2). In support of this hypothesis, Matrin3 was found to interact with itself in a yeast two hybrid screen ${ }^{27}$. Another striking feature is that the introns flanking the Matrin3 repressed exons are significantly longer than those flanking other cassette exons, including those regulated by PTBP1. Longer introns may be required to prevent the "bind and spread" mode of action from affecting the flanking constitutive splice sites.

Notably, both of the Matrin3-related proteins RBM20 and ZNF638 have also been reported to regulate alternative splicing ${ }^{32,35,36}$, suggesting that it is a core function of this group of proteins. RBM20 has similar RNA binding preference to PTBP1 and Matrin3, consistent with the similarity between their RRMs (Fig 1). Strikingly, RBM20 appears to act primarily as a splicing repressor and like Matrin3 binds within both introns flanking repressed exons ${ }^{32}$. Despite these similarities, there are interesting contrasts. While Matrin3 interacts with a known splicing repressor PTBP1 via its PRI, RBM20 interacts with a number of core splicing factors, consistent with having an RS domain, as well as with hnRNPs ${ }^{32}$.

\section{Matrin3 function in RNA stability}

Steady-state RNA levels are determined by the rates of RNA synthesis and degradation. In addition to its roles in transcription, Matrin3 has also been implicated the stability of a number of $m R N A s^{24}$. Since Matrin3 interacts with several transcription associated factors, such as DHX9 and hnRNPK, and the noncoding small RNA 7SK, the authors examined alterations in gene expression upon Matrin3 depletion in U2OS cells. Matrin3 depletion decreases the mRNA levels of 77 genes, and Matrin3 binding to these mRNAs was indicated by RNA immunoprecipitation. 
This interaction is dependent on the second RRM, which interestingly is also the one involved in the RNA-dependent interaction with DHX9 and hnRNPK. The reduced levels of these mRNAs were shown to be due to reduced RNA stability rather than transcriptional down-regulation, indicated by reduced mRNA half-life of three tested mRNAs ${ }^{24}$. The exact molecular mechanism of Matrin3-dependent mRNA stabilization is not entirely understood. However, a subset of stabilized mRNAs might be explained by nonsense mediated decay (NMD) linked to alternative splicing (ASNMD $)^{19}$. These are splicing events in which one of the splicing patterns introduces a premature termination codon (PTC) leading to $\mathrm{NMD}^{51}$. Analysis of mRNA level and splicing changes upon Matrin3 knockdown showed that 15 out of 22 genes with changes in expression greater than 1.5 fold had an associated AS-NMD event that might explain the changes in mRNA level ${ }^{19}$.

\section{Matrin3 function in viral biology}

Viruses exploit their host systems to serve their own interests in several steps of their life cycle. Generation of infectious HIV-1 viral progeny requires the cytoplasmic export of several spliced and unspliced subgenomic HIV-1 mRNAs. The Rev protein is crucial in HIV-1 biogenesis and is produced by the fully spliced RNA, and in turn Rev facilitates the export of incompletely spliced RNAs that will express the other essential HIV-1 components, and ultimately genomic RNA for assembly of new HIV1 particles. Rev binds to the Rev Response Elements (RRE) present in the RNA and together with host components it can escape the nucleus and lead the RNAs to the ribosome for expression of viral proteins (Reviewed $\mathrm{in}^{52}$ ). Two parallel studies using different approaches identified Matrin3 as a regulator of HIV-1 expression: one by RNA-affinity isolation of proteins interacting with the HIV-1 RNA and the other by yeast two hybrid screen using PTBP1 as a bait, as this splicing factor has been shown to affect HIV-1 gene expression ${ }^{53,54}$. Manipulation of Matrin3 levels greatly affected the activity of Rev, indicative of a role of Matrin3 as a Rev co-regulator. Rev, Matrin3 and RRE-containing RNAs together form a complex suggesting that RNA bridges the Rev-Matrin3 interaction, and supporting this is the requirement for Matrin3's RRMs for this activity. Here, as in some of the other Matrin3 associated functions, PSF also plays a role ${ }^{55}$. However their RNA association is only partially 
overlapping. PSF is recruited to the RRE-containing RNA along with Rev cotranscriptionally and is thought to be released before RNA accumulation at the nuclear matrix or its export, while Matrin3 is recruited post-transcriptionally and remains with the RNA in the nuclear matrix until its export. Viral use of Matrin3 is possibly not restricted to HIV-1, and accordingly a kinase encoded by alphaherpesvirus ORF66 targets predominantly Matrin3, modifying threonine 150, which is required to avoid cytoplasmic aggregates of Matrin3 in later stages of infection similar to what has been observed with the lamins ${ }^{56,57}$.

Along with PSF and NONO, Matrin3 was also identified as a component of a complex involved in nuclear retention of RNA with long double-stranded regions, possibly associated with adenosine to inosine editing ${ }^{58}$. This could be of interest as a possible cellular antiviral mechanism. However, subsequent analyses suggest that this resulted from nuclear paraspeckle localization mediated by PSF and NONO, with no evidence for involvement of Matrin $3^{59}$.

\section{Matrin3 function in cell survival and DNA damage response}

The importance of the multiple functions of Matrin3 is evident from the dramatic effect of its depletion on cell proliferation and on the ability of cells to respond to DNA damaging compounds ${ }^{17,60,61}$. Matrin3 is involved in the response to several signals, such as the activation of NMDA receptors ${ }^{62}$ or the induction of DNA double strand breaks ${ }^{60}$. Both lead to post-translation modification of Matrin3 by phosphorylation at serine residues, with the former mediated by protein kinase $A$ (PKA) and the latter by the kinase ataxia-telangiectasia mutated (ATM). PKA targets mainly a serine at position $188^{57}$ while ATM targets serine $208^{60}$. The consequences of these modifications are quite distinct. Matrin3 is the main PKA target upon NMDA receptor activation in rat brain, which culminates in Matrin3 degradation and neuronal cell death, effects that can be blocked by PKA inhibitors ${ }^{62}$. Several caspases, typically activated/released during apoptosis, can cleave Matrin3, although it is not clear if this is the mechanism occurring during PKA-mediated degradation ${ }^{63}$. Down-regulation of Matrin3 by siRNA transfection in endothelial cells culminates in a decrease of cell proliferation followed by cell necrosis ${ }^{61}$. This 
suggests that although Matrin3 down-regulation is involved in the regulated apoptotic death response to NMDA signalling, it is not sufficient and that in the absence of signalling Matrin3 depletion alone leads to necrotic cell death. The exact mechanism by which Matrin3 ensures proper cell proliferation is not clear, although it most likely will be related to its nuclear functions as it requires its nuclear localisation ${ }^{17}$.

Persistent DNA damage can lead to cell death, and Matrin3 can function in several steps ensuring that only undamaged cells escape cell death, although no direct links between its role in DNA damage response and cell death have yet been reported. DNA double strand breaks (DDB) trigger ATM mediated phosphorylation of Matrin3 at serine 208, and this seems to be required for the DNA damage response ${ }^{60}$. This response involves activation of a cell cycle checkpoint that stalls cells to ensure sufficient time for DNA repair. Matrin3 can form a complex with proteins involved in DNA damage response, PSF and NONO, as well as the DNA binding proteins Ku80/Ku70, independent of any DNA damage. PSF and NONO are recruited to sites of DNA damage, and while Matrin3 itself is neither recruited nor affects the recruitment of other components, it does significantly alter the retention time of these proteins at the damage sites. This suggests that Matrin3 is involved in the early steps of the DNA damage response and is required for the dynamic recruitment and release of PSF/NONO from the damage site, although it is itself not recruited to the site ${ }^{60}$.

\section{Associations of Matrin3 with human diseases}

In addition to its effect on viruses such as HIV1, inherited mutations of Matrin3 have also been shown to be associated with the neurodegenerative disease amyotrophic lateral sclerosis (ALS). ALS is a neurodegenerative disease affecting lower and upper motor neurons including the spinal cord, typically with adult onset. A Ser85Cys mutation in Matrin3 was found in 1998 as the autosomal dominant mutation in a family with multiple members diagnosed with myopathy ${ }^{64}$. Subsequently, a Phe115Cys mutation in Matrin3 was found to segregate with disease in a Sardinian family suffering from slow progressing ALS, and re-evaluation of the original family established that the Ser85Cys mutations also causes ALS ${ }^{65}$. Matrin3 mutations are rare in ALS, since in 96 patients with sporadic disease only 
one missense mutation was found in Matrin3. Patients with Matrin3 Ser85Cys or Phe115Cys mutation suffer from brisk knee reflexes and a 'split-hand' pattern of weakness $^{65,66}$, symptoms also observed in other ALS patients.

At the molecular level, Phe115Cys mutation leads to intra-nuclear inclusion, and a pronounced increase in Matrin3 protein abundance in the spinal cord neurons. Aggregation of Matrin3 is also seen in ALS patients with the more common C9orf72 tri-nucleotide expansion, suggesting that aggregate formation of Matrin3 (and other RBPs) is widespread in ALS patients ${ }^{65}$. This phenotype is highly similar to other RBPs in which mutations lead to aggregation and manifestation of ALS or related motor neuron diseases, including TDP43, FUS, hnRNPA1 and hnRNPA2 ${ }^{67}$. The basis for pathology could be the loss of function of the protein(s), which may lead to deregulated alternative splicing. Alternatively, the aggregates may actively disrupt cellular functions via a toxic gain of function. The two mechanisms are not mutually exclusive and evidence has been presented for both in the cases of other RNA binding proteins such as TDP43 and FUS ${ }^{68}$.

As noted above, expression of the Matrin3-related protein RBM20 is restricted to the heart and is especially high in early development. Consistent with this, mutations of RBM20 were identified in 8 independent families associated with dilated cardiomyopathy ${ }^{69,70}$. The missense mutations were all located in a hotspot corresponding to the arginine-serine domain of RBM20. Moreover, a large deletion of the RBM20 gene was found to be responsible for altered splicing of titin and other mRNAs in a rat model that also showed symptoms of dilated cardiomyopathy ${ }^{35}$. This suggests that the cardiomyopathy results from missplicing associated with loss of RBM20 function. It remains to be determined whether the mechanisms of ALS pathology arising from Matrin3 mutations are similar to the cardiomyopathy arising from RBM20 mutations.

\section{Conclusions and perspectives}

Evidence of the multiple functions of Matrin3 has accumulated over the last 25 years since it was discovered as a component of nuclear matrix. The full extent of functional coupling between the different stages of gene expression has also come into focus during this period, and like many other proteins, Matrin3 has clear roles at 
numerous levels of gene expression. While it seems obvious that Matrin3 function is linked to the nuclear matrix, the extent to which its involvement in processes from transcriptional activation and pre-mRNA splicing to export of viral RNAs, requires anchoring of RNPs to the nuclear matrix remains unresolved. A precise mutation to specifically disrupt matrix localization would be an invaluable tool in this regard. Matrin3 function might in part be attributed to limiting diffusion of several co-factors, as suggested by the change in retention time of PSF and NONO upon DNA damage ${ }^{60}$. Much remains to be learned about the molecular interactions and cellular roles of Matrin3 and its related proteins, RBM20 and ZNF638, as well as the molecular basis of pathologies arising from their malfunction. The pace of discoveries about Matrin3 has accelerated over the past few years, with new insights emerging from several directions, including investigations of viral mechanisms and of diseases of motor neurons and the heart. We anticipate that Matrin3 will become a prime exemplar of the multiple ways in which RNP functions are inter-linked with genome organisation and the nuclear matrix.

\section{Acknowledgments}

We thank Clare Gooding and Dipen Rajgor for critical comments on the manuscript. Work in the CWJS lab on Matrin3 is funded by a grant from the Wellcome Trust (092900). JA was funded by a Boehringer Ingelheim Fonds studentship. 


\section{Figure Legends}

\section{Figure 1. Domain organization of Matrin3 and related proteins}

A) Schematic diagram of structural organization of Matrin3 (middle), ZNF638 (top) and RBM20 (bottom). The percent sequence identity between the RRM and ZF domains of Matrin3 compared to ZNF638 and RBM20 is indicated. Disease - associated aminoacid residues S85, F115, P154 and T622, and the phosphorylation sites at S188 and S208, are indicated.

B) Relative expression of Matrin3, RBM20 and ZNF638 across human tissues. Darker red correlates with high expression levels and light red with low expression. Data taken from The Human Protein Atlas ${ }^{71}$.

\section{Figure 2. Proposed model for splicing regulation by Matrin3}

Cartoon depicting an exon not regulated by Matrin3 $(A)$ and one regulated by Matrin3 (B). The presence of Matrin3 binding site ( $\mathrm{B}$ - boxed sequence) on the introns flanking the exon recruits the Matrin3 molecules, which can be bound or not to other splicing regulators like PTBP1. 
Table 1 - Matrin3 interacting proteins

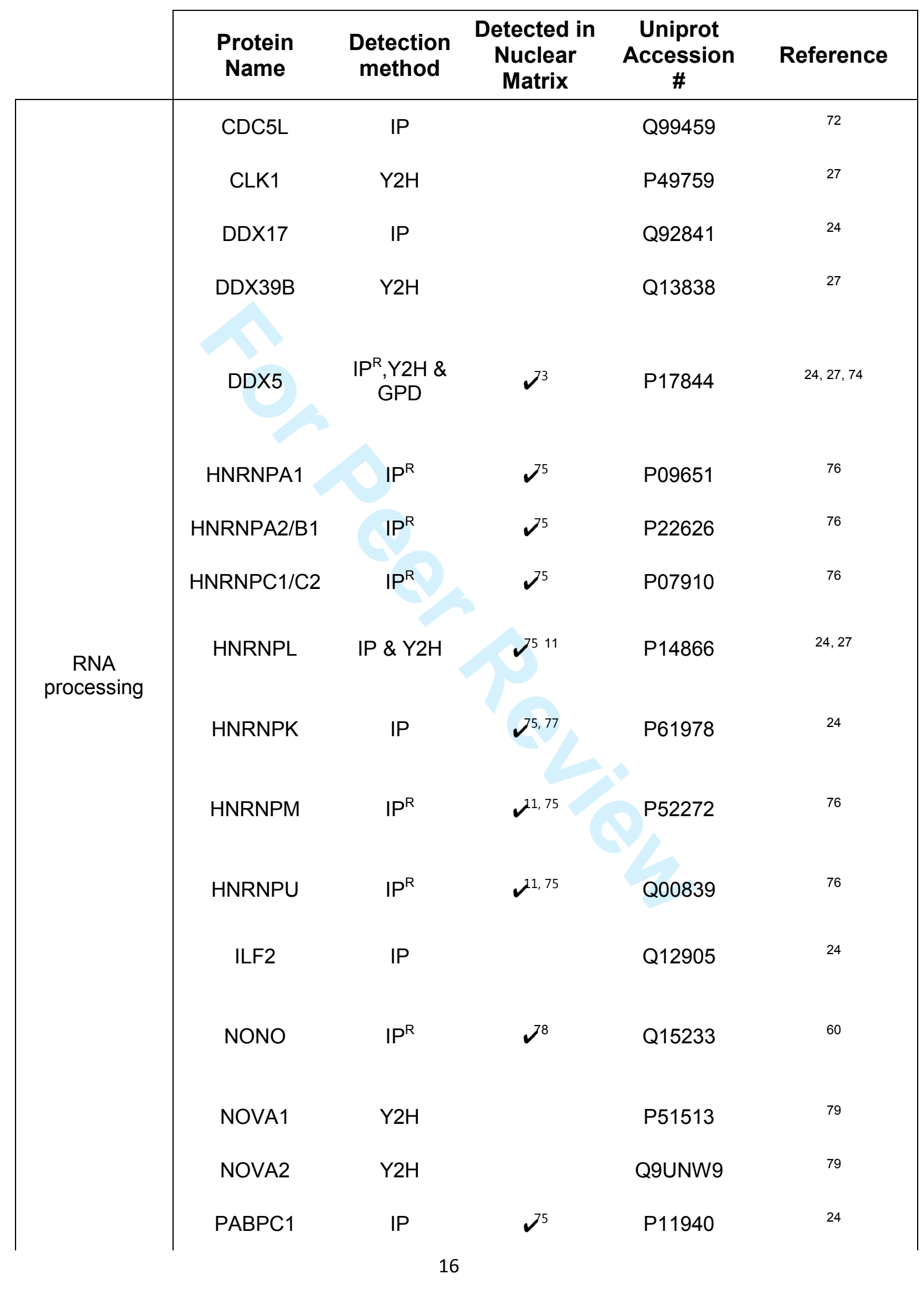




\begin{tabular}{|c|c|c|c|c|c|}
\hline & PCBP2 & $\mathrm{Y} 2 \mathrm{H}$ & & Q15366 & 27 \\
\hline & PTBP1 & $I^{R} \& G P D$ & $\lambda^{578}$ & P26599 & 19,24 \\
\hline & РTBP2 & $\mathrm{Y} 2 \mathrm{H}$ & & Q9UKA9 & 80 \\
\hline & РTBP3 & $I^{R}$ & & O95758 & 81 \\
\hline & RBMX & $\mathrm{Y} 2 \mathrm{H}$ & & P38159 & 27 \\
\hline & PSF & $I P^{R}$ & $J^{8}$ & P23246 & 6055 \\
\hline & SFRS7 & $\mathrm{Y} 2 \mathrm{H}$ & $\lambda^{11}$ & Q16629 & 27 \\
\hline & SRPK2 & $\mathrm{Y} 2 \mathrm{H}$ & & P78362 & 27 \\
\hline & $\begin{array}{c}\text { TARDBP/TDP4 } \\
3\end{array}$ & IP & & Q13148 & $\begin{array}{c}\text { Freibaum et al, } \\
2010\end{array}$ \\
\hline \multirow{12}{*}{ Transcription } & DDX3X & $I P^{R}$ & $\nu^{11}$ & O00571 & 76 \\
\hline & DHX9 & IP & $\nu^{1}$ & Q08211 & 24 \\
\hline & ERG & IP \& GPD & & P11308 & 82 \\
\hline & FOXG1B & $\mathrm{Y} 2 \mathrm{H}$ & & P55316 & 27 \\
\hline & NFX1 & IP & & Q12986 & 83 \\
\hline & NR2F1 & $\mathrm{Y} 2 \mathrm{H}$ & & P10589 & 27 \\
\hline & RGS6 & $\mathrm{Y} 2 \mathrm{H}$ & & P49758 & 27 \\
\hline & PoU1F1/Pit1 & IP & $\boldsymbol{\nu}^{39}$ & P28069 & 39 \\
\hline & POLR2A & IP & $\sigma^{6}$ & P24928 & 84 \\
\hline & RPAP1 & Y2H & & Q9BWH6 & 27 \\
\hline & SAFB & $\mathrm{Y} 2 \mathrm{H}$ & 85 & Q15424 & 27 \\
\hline & SLTM & $\mathrm{Y} 2 \mathrm{H}$ & $\boldsymbol{\nu}^{11}$ & Q9NWH9 & 27 \\
\hline
\end{tabular}




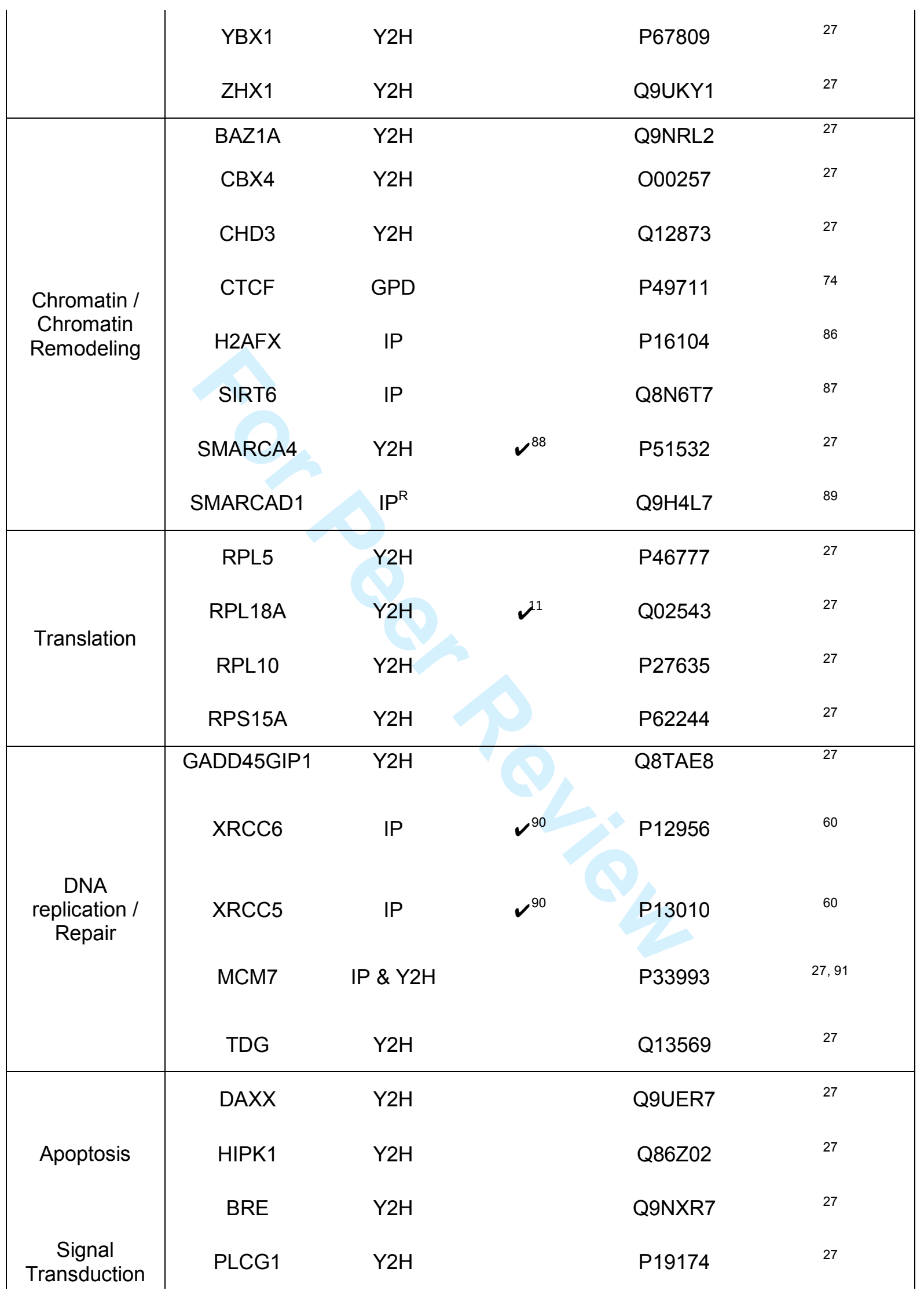


1

2

3

4

5

6

7

8

9

10

11

12

13

14

15

16

17

18

19

20

21

22

23

24

25

26

27

28

28
29

30

31

32

33

34

35

36

37

38

39

40

41

42

43

44

45

46

47

48

49

50

51

52

53

54

55

56

57

58

59

60
19

\begin{tabular}{|c|c|c|c|c|c|}
\hline & CIB1 & $\mathrm{Y} 2 \mathrm{H}$ & & Q99828 & 27 \\
\hline $\begin{array}{c}\text { Nuclear } \\
\text { Organization }\end{array}$ & LMNA & $I P^{R} \& G P D$ & $\boldsymbol{\lambda}^{1,11}$ & P02545 & 26 \\
\hline
\end{tabular}

$\mathrm{Y} 2 \mathrm{H}$ - Yeast two hybrid; IP - Immunoprecipitation; GPD - GST pulldown, ${ }^{\mathrm{R}}$ - Ribonuclease resistant interaction 


\section{References}

1. Nakayasu H, Berezney R. Nuclear matrins: identification of the major nuclear matrix proteins. Proc Natl Acad Sci U S A 1991, 88:10312-10316.

2. Belgrader P, Dey R, Berezney R. Molecular cloning of matrin 3. A 125-kilodalton protein of the nuclear matrix contains an extensive acidic domain. J Biol Chem 1991, 266:9893-9899.

3. Bode J, Goetze S, Heng H, Krawetz SA, Benham C. From DNA structure to gene expression: mediators of nuclear compartmentalization and dynamics. Chromosome Res 2003, 11:435445.

4. Martins RP, Ostermeier GC, Krawetz SA. Nuclear matrix interactions at the human protamine domain: a working model of potentiation. J Biol Chem 2004, 279:51862-51868.

5. Linnemann AK, Platts AE, Krawetz SA. Differential nuclear scaffold/matrix attachment marks expressed genes. Hum Mol Genet 2009, 18:645-654.

6. Mortillaro MJ, Blencowe BJ, Wei X, Nakayasu H, Du L, Warren SL, Sharp PA, Berezney R. A hyperphosphorylated form of the large subunit of RNA polymerase II is associated with splicing complexes and the nuclear matrix. Proc Natl Acad Sci U S A 1996, 93:8253-8257.

7. Yusufzai TM, Felsenfeld G. The 5'-HS4 chicken beta-globin insulator is a CTCF-dependent nuclear matrix-associated element. Proc Natl Acad Sci U S A 2004, 101:8620-8624.

8. Mesner LD, Hamlin JL, Dijkwel PA. The matrix attachment region in the Chinese hamster dihydrofolate reductase origin of replication may be required for local chromatid separation. Proc Natl Acad Sci U S A 2003, 100:3281-3286.

9. Nickerson J. Experimental observations of a nuclear matrix. J Cell Sci 2001, 114:463-474.

10. $\mathrm{MaH}$, Siegel AJ, Berezney R. Association of chromosome territories with the nuclear matrix. Disruption of human chromosome territories correlates with the release of a subset of nuclear matrix proteins. J Cell Biol 1999, 146:531-542.

11. Engelke R, Riede J, Hegermann J, Wuerch A, Eimer S, Dengjel J, Mittler G. The quantitative nuclear matrix proteome as a biochemical snapshot of nuclear organization. $J$ Proteome Res 2014, 13:3940-3956.

12. Pederson T. Half a century of "the nuclear matrix". Mol Biol Cell 2000, 11:799-805.

13. Razin SV, larovaia OV, Vassetzky YS. A requiem to the nuclear matrix: from a controversial concept to 3D organization of the nucleus. Chromosoma 2014, 123:217-224.

14. Hibino Y, Nakamura K, Tsukada S, Sugano N. Purification and characterization of nuclear scaffold proteins which bind to a highly repetitive bent DNA from rat liver. Biochim Biophys Acta 1993, 1174:162-170.

15. Hibino Y, Tsukada S, Sugano N. Properties of a DNA-binding protein from rat nuclear scaffold fraction. Biochem Biophys Res Commun 1993, 197:336-342.

16. Hibino Y, Usui T, Morita Y, Hirose N, Okazaki M, Sugano N, Hiraga K. Molecular properties and intracellular localization of rat liver nuclear scaffold protein P130. Biochim Biophys Acta 2006, 1759:195-207.

17. Hisada-Ishii S, Ebihara M, Kobayashi N, Kitagawa Y. Bipartite nuclear localization signal of matrin 3 is essential for vertebrate cells. Biochem Biophys Res Commun 2007, 354:72-76.

18. Dinkel H, Van Roey K, Michael S, Davey NE, Weatheritt RJ, Born D, Speck T, Kruger D, Grebnev G, Kuban M, et al. The eukaryotic linear motif resource ELM: 10 years and counting. Nucleic Acids Res 2014, 42:D259-266. 
19. Coelho MB, Attig J, Bellora N, Konig J, Hallegger M, Kayikci M, Eyras E, Ule J, Smith CW. Nuclear matrix protein Matrin3 regulates alternative splicing and forms overlapping regulatory networks with PTB. EMBO J 2015, 34:653-668.

20. Hakes DJ, Berezney R. DNA binding properties of the nuclear matrix and individual nuclear matrix proteins. Evidence for salt-resistant DNA binding sites. J Biol Chem 1991, 266:1113111140.

21. Muto Y, Pomeranz Krummel D, Oubridge C, Hernandez H, Robinson CV, Neuhaus D, Nagai K. The structure and biochemical properties of the human spliceosomal protein U1C. J Mol Biol 2004, 341:185-198.

22. Ray D, Kazan H, Cook KB, Weirauch MT, Najafabadi HS, Li X, Gueroussov S, Albu M, Zheng H, Yang A, et al. A compendium of RNA-binding motifs for decoding gene regulation. Nature 2013, 499:172-177.

23. Clery A, Blatter M, Allain FH. RNA recognition motifs: boring? Not quite. Curr Opin Struct Biol 2008, 18:290-298.

24. Salton M, Elkon R, Borodina T, Davydov A, Yaspo ML, Halperin E, Shiloh Y. Matrin 3 binds and stabilizes mRNA. PLoS One 2011, 6:e23882.

25. Witten JT, Ule J. Understanding splicing regulation through RNA splicing maps. Trends Genet 2011, 27:89-97.

26. Depreux FF, Puckelwartz MJ, Augustynowicz A, Wolfgeher D, Labno CM, Pierre-Louis D, Cicka D, Kron SJ, Holaska J, McNally EM. Disruption of the lamin A and matrin-3 interaction by myopathic LMNA mutations. Hum Mol Genet 2015, 24:4284-4295.

27. Zeitz MJ, Malyavantham KS, Seifert B, Berezney R. Matrin 3: chromosomal distribution and protein interactions. J Cell Biochem 2009, 108:125-133.

28. Zeng C, van Wijnen AJ, Stein JL, Meyers S, Sun W, Shopland L, Lawrence JB, Penman S, Lian $J B$, Stein GS, et al. Identification of a nuclear matrix targeting signal in the leukemia and bone-related AML/CBF-alpha transcription factors. Proc Natl Acad Sci U S A 1997, 94:67466751.

29. Seo J, Lozano MM, Dudley JP. Nuclear matrix binding regulates SATB1-mediated transcriptional repression. J Biol Chem 2005, 280:24600-24609.

30. Yarosh CA, lacona JR, Lutz CS, Lynch KW. PSF: nuclear busy-body or nuclear facilitator? Wiley Interdiscip Rev RNA 2015, 6:351-367.

31. Inagaki H, Matsushima Y, Nakamura K, Ohshima M, Kadowaki T, Kitagawa Y. A large DNAbinding nuclear protein with RNA recognition motif and serine/arginine-rich domain. J Biol Chem 1996, 271:12525-12531.

32. Maatz H, Jens M, Liss M, Schafer S, Heinig M, Kirchner M, Adami E, Rintisch C, Dauksaite V, Radke $\mathrm{MH}$, et al. RNA-binding protein RBM20 represses splicing to orchestrate cardiac premRNA processing. J Clin Invest 2014, 124:3419-3430.

33. Perez I, Lin CH, McAfee JG, Patton JG. Mutation of PTB binding sites causes misregulation of alternative 3' splice site selection in vivo. RNA 1997, 3:764-778.

34. Oberstrass FC, Auweter SD, Erat M, Hargous $Y$, Henning A, Wenter P, Reymond L, AmirAhmady B, Pitsch S, Black DL, et al. Structure of PTB bound to RNA: specific binding and implications for splicing regulation. Science 2005, 309:2054-2057.

35. Guo W, Schafer S, Greaser ML, Radke MH, Liss M, Govindarajan T, Maatz H, Schulz H, Li S, Parrish AM, et al. RBM20, a gene for hereditary cardiomyopathy, regulates titin splicing. Nat Med 2012, 18:766-773.

36. Du C, Ma X, Meruvu S, Hugendubler L, Mueller E. The adipogenic transcriptional cofactor ZNF638 interacts with splicing regulators and influences alternative splicing. J Lipid Res 2014, 55:1886-1896.

37. Hibino $Y$, Ohzeki $H$, Sugano $N$, Hiraga K. Transcription modulation by a rat nuclear scaffold protein, $\mathrm{P} 130$, and a rat highly repetitive DNA component or various types of animal and 
plant matrix or scaffold attachment regions. Biochem Biophys Res Commun 2000, 279:282287.

38. Hibino $\mathrm{Y}$, Ohzeki $\mathrm{H}$, Hirose $\mathrm{N}$, Morita $\mathrm{Y}$, Sugano N. Involvement of DNA methylation in binding of a highly repetitive DNA component to nuclear scaffold proteins from rat liver. Biochem Biophys Res Commun 1998, 252:296-301.

39. Skowronska-Krawczyk D, Ma Q, Schwartz M, Scully K, Li W, Liu Z, Taylor H, Tollkuhn J, Ohgi $K A$, Notani $D$, et al. Required enhancer-matrin-3 network interactions for a homeodomain transcription program. Nature 2014, 514:257-261.

40. Cook PR. The organization of replication and transcription. Science 1999, 284:1790-1795.

41. Guelen L, Pagie L, Brasset E, Meuleman W, Faza MB, Talhout W, Eussen BH, de Klein A, Wessels $L$, de Laat $W$, et al. Domain organization of human chromosomes revealed by mapping of nuclear lamina interactions. Nature 2008, 453:948-951.

42. Martens JH, Verlaan M, Kalkhoven E, Dorsman JC, Zantema A. Scaffold/matrix attachment region elements interact with a p300-scaffold attachment factor $A$ complex and are bound by acetylated nucleosomes. Mol Cell Biol 2002, 22:2598-2606.

43. Hall LL, Carone DM, Gomez AV, Kolpa HJ, Byron M, Mehta N, Fackelmayer FO, Lawrence JB. Stable COT-1 repeat RNA is abundant and is associated with euchromatic interphase chromosomes. Cell 2014, 156:907-919.

44. Malyavantham KS, Bhattacharya S, Barbeitos M, Mukherjee L, Xu J, Fackelmayer FO, Berezney R. Identifying functional neighborhoods within the cell nucleus: proximity analysis of early S-phase replicating chromatin domains to sites of transcription, RNA polymerase II, HP1gamma, matrin 3 and SAF-A. J Cell Biochem 2008, 105:391-403.

45. Nilsen TW, Graveley BR. Expansion of the eukaryotic proteome by alternative splicing. Nature 2010, 463:457-463.

46. Rideau AP, Gooding C, Simpson PJ, Monie TP, Lorenz M, Huttelmaier S, Singer RH, Matthews $S$, Curry S, Smith CW. A peptide motif in Raver1 mediates splicing repression by interaction with the PTB RRM2 domain. Nat Struct Mol Biol 2006, 13:839-848.

47. Joshi A, Coelho MB, Kotik-Kogan O, Simpson PJ, Matthews SJ, Smith CW, Curry S. Crystallographic analysis of polypyrimidine tract-binding protein-Raver1 interactions involved in regulation of alternative splicing. Structure 2011, 19:1816-1825.

48. Gueroussov S, Gonatopoulos-Pournatzis T, Irimia M, Raj B, Lin ZY, Gingras AC, Blencowe BJ. RNA SPLICING. An alternative splicing event amplifies evolutionary differences between vertebrates. Science 2015, 349:868-873.

49. Robinson $\mathrm{F}$, Smith CW. A splicing repressor domain in polypyrimidine tract-binding protein. J Biol Chem 2006, 281:800-806.

50. Llorian M, Schwartz S, Clark TA, Hollander D, Tan LY, Spellman R, Gordon A, Schweitzer AC, de la Grange $P$, Ast $G$, et al. Position-dependent alternative splicing activity revealed by global profiling of alternative splicing events regulated by PTB. Nat Struct Mol Biol 2010, 17:1114-1123.

51. McGlincy NJ, Smith CW. Alternative splicing resulting in nonsense-mediated mRNA decay: what is the meaning of nonsense? Trends Biochem Sci 2008, 33:385-393.

52. Cullen BR. Viral RNAs: lessons from the enemy. Cell 2009, 136:592-597.

53. Kula A, Guerra J, Knezevich A, Kleva D, Myers MP, Marcello A. Characterization of the HIV-1 RNA associated proteome identifies Matrin 3 as a nuclear cofactor of Rev function.

Retrovirology 2011, 8:60.

54. Yedavalli VS, Jeang KT. Matrin 3 is a co-factor for HIV-1 Rev in regulating post-transcriptional viral gene expression. Retrovirology 2011, 8:61.

55. Kula A, Gharu L, Marcello A. HIV-1 pre-mRNA commitment to Rev mediated export through PSF and Matrin 3. Virology 2013, 435:329-340. 
56. Mou F, Forest T, Baines JD. US3 of herpes simplex virus type 1 encodes a promiscuous protein kinase that phosphorylates and alters localization of lamin A/C in infected cells. $J$ Virol 2007, 81:6459-6470.

57. Erazo A, Yee MB, Banfield BW, Kinchington PR. The alphaherpesvirus US3/ORF66 protein kinases direct phosphorylation of the nuclear matrix protein matrin 3. J Virol 2011, 85:568581.

58. Zhang Z, Carmichael GG. The fate of dsRNA in the nucleus: a p54(nrb)-containing complex mediates the nuclear retention of promiscuously A-to-I edited RNAs. Cell 2001, 106:465-475.

59. Chen LL, Carmichael GG. Altered nuclear retention of mRNAs containing inverted repeats in human embryonic stem cells: functional role of a nuclear noncoding RNA. Mol Cell 2009, 35:467-478.

60. Salton M, Lerenthal Y, Wang SY, Chen DJ, Shiloh Y. Involvement of Matrin 3 and SFPQ/NONO in the DNA damage response. Cell Cycle 2010, 9:1568-1576.

61. Przygodzka P, Boncela J, Cierniewski CS. Matrin 3 as a key regulator of endothelial cell survival. Exp Cell Res 2011, 317:802-811.

62. Giordano G, Sanchez-Perez AM, Montoliu C, Berezney R, Malyavantham K, Costa LG, Calvete $\mathrm{JJ}$, Felipo V. Activation of NMDA receptors induces protein kinase A-mediated phosphorylation and degradation of matrin 3. Blocking these effects prevents NMDAinduced neuronal death. J Neurochem 2005, 94:808-818.

63. Valencia CA, Ju W, Liu R. Matrin 3 is a Ca2+/calmodulin-binding protein cleaved by caspases. Biochem Biophys Res Commun 2007, 361:281-286.

64. Feit H, Silbergleit A, Schneider LB, Gutierrez JA, Fitoussi RP, Reyes C, Rouleau GA, Brais B, Jackson CE, Beckmann JS, et al. Vocal cord and pharyngeal weakness with autosomal dominant distal myopathy: clinical description and gene localization to 5q31. Am J Hum Genet 1998, 63:1732-1742.

65. Johnson JO, Pioro EP, Boehringer A, Chia R, Feit H, Renton AE, Pliner HA, Abramzon Y, Marangi G, Winborn BJ, et al. Mutations in the Matrin 3 gene cause familial amyotrophic lateral sclerosis. Nat Neurosci 2014, 17:664-666.

66. Yamashita S, Ando Y. Genotype-phenotype relationship in hereditary amyotrophic lateral sclerosis. Transl Neurodegener 2015, 4:13.

67. Kim HJ, Kim NC, Wang YD, Scarborough EA, Moore J, Diaz Z, MacLea KS, Freibaum B, Li S, Molliex A, et al. Mutations in prion-like domains in hnRNPA2B1 and hnRNPA1 cause multisystem proteinopathy and ALS. Nature 2013, 495:467-473.

68. Mackenzie IR, Rademakers R, Neumann M. TDP-43 and FUS in amyotrophic lateral sclerosis and frontotemporal dementia. Lancet Neurol 2010, 9:995-1007.

69. Brauch KM, Karst ML, Herron KJ, de Andrade M, Pellikka PA, Rodeheffer RJ, Michels VV, Olson TM. Mutations in ribonucleic acid binding protein gene cause familial dilated cardiomyopathy. J Am Coll Cardiol 2009, 54:930-941.

70. Linke WA, Bucker S. King of hearts: a splicing factor rules cardiac proteins. Nat Med 2012, 18:660-661.

71. Uhlen M, Fagerberg L, Hallstrom BM, Lindskog C, Oksvold P, Mardinoglu A, Sivertsson A, Kampf C, Sjostedt E, Asplund A, et al. Proteomics. Tissue-based map of the human proteome. Science 2015, 347:1260419.

72. Lleres D, Denegri M, Biggiogera M, Ajuh P, Lamond Al. Direct interaction between hnRNP-M and CDC5L/PLRG1 proteins affects alternative splice site choice. EMBO Rep 2010, 11:445451.

73. Akileswaran L, Taraska JW, Sayer JA, Gettemy JM, Coghlan VM. A-kinase-anchoring protein AKAP95 is targeted to the nuclear matrix and associates with p68 RNA helicase. J Biol Chem 2001, 276:17448-17454.

74. Fujita T, Fujii H. Direct identification of insulator components by insertional chromatin immunoprecipitation. PLoS One 2011, 6:e26109. 
75. Mattern KA, Humbel BM, Muijsers AO, de Jong L, van Driel R. hnRNP proteins and B23 are the major proteins of the internal nuclear matrix of HeLa S3 cells. J Cell Biochem 1996, 62:275-289.

76. Erazo A, Goff SP. Nuclear matrix protein Matrin 3 is a regulator of ZAP-mediated retroviral restriction. Retrovirology 2015, 12:57.

77. Samuel SK, Spencer VA, Bajno L, Sun JM, Holth LT, Oesterreich S, Davie JR. In situ crosslinking by cisplatin of nuclear matrix-bound transcription factors to nuclear DNA of human breast cancer cells. Cancer Res 1998, 58:3004-3008.

78. Meissner M, Dechat T, Gerner C, Grimm R, Foisner R, Sauermann G. Differential nuclear localization and nuclear matrix association of the splicing factors PSF and PTB. J Cell Biochem 2000, 76:559-566.

79. Polydorides AD, Okano HJ, Yang YY, Stefani G, Darnell RB. A brain-enriched polypyrimidine tract-binding protein antagonizes the ability of Nova to regulate neuron-specific alternative splicing. Proc Natl Acad Sci U S A 2000, 97:6350-6355.

80. Hegele A, Kamburov A, Grossmann A, Sourlis C, Wowro S, Weimann M, Will CL, Pena V, Luhrmann R, StelzI U. Dynamic protein-protein interaction wiring of the human spliceosome. Mol Cell 2012, 45:567-580.

81. Brazao TF, Demmers J, van IW, Strouboulis J, Fornerod M, Romao L, Grosveld FG. A new function of ROD1 in nonsense-mediated mRNA decay. FEBS Lett 2012, 586:1101-1110.

82. Wang S, Kollipara RK, Srivastava N, Li R, Ravindranathan P, Hernandez E, Freeman E, Humphries CG, Kapur P, Lotan Y, et al. Ablation of the oncogenic transcription factor ERG by deubiquitinase inhibition in prostate cancer. Proc Natl Acad Sci U S A 2014, 111:4251-4256.

83. Katzenellenbogen RA, Egelkrout EM, Vliet-Gregg P, Gewin LC, Gafken PR, Galloway DA. NFX1-123 and poly(A) binding proteins synergistically augment activation of telomerase in human papillomavirus type 16 E6-expressing cells. J Virol 2007, 81:3786-3796.

84. Das R, Yu J, Zhang Z, Gygi MP, Krainer AR, Gygi SP, Reed R. SR proteins function in coupling RNAP II transcription to pre-mRNA splicing. Mol Cell 2007, 26:867-881.

85. Oesterreich S, Lee AV, Sullivan TM, Samuel SK, Davie JR, Fuqua SA. Novel nuclear matrix protein HET binds to and influences activity of the HSP27 promoter in human breast cancer cells. J Cell Biochem 1997, 67:275-286.

86. Yang X, Zou P, Yao J, Yun D, Bao H, Du R, Long J, Chen X. Proteomic dissection of cell typespecific $\mathrm{H} 2 \mathrm{AX}$-interacting protein complex associated with hepatocellular carcinoma. J Proteome Res 2010, 9:1402-1415.

87. Simeoni F, Tasselli L, Tanaka S, Villanova L, Hayashi M, Kubota K, Isono F, Garcia BA, Michishita-Kioi E, Chua KF. Proteomic analysis of the SIRT6 interactome: novel links to genome maintenance and cellular stress signaling. Sci Rep 2013, 3:3085.

88. Reyes JC, Muchardt C, Yaniv M. Components of the human SWI/SNF complex are enriched in active chromatin and are associated with the nuclear matrix. J Cell Biol 1997, 137:263-274.

89. Rowbotham SP, Barki L, Neves-Costa A, Santos F, Dean W, Hawkes N, Choudhary P, Will WR, Webster J, Oxley $D$, et al. Maintenance of silent chromatin through replication requires SWI/SNF-like chromatin remodeler SMARCAD1. Mol Cell 2011, 42:285-296.

90. Yu E, Song K, Moon H, Maul GG, Lee I. Characteristic immunolocalization of Ku protein as nuclear matrix. Hybridoma 1998, 17:413-420.

91. Huang TH, Huo L, Wang YN, Xia W, Wei Y, Chang SS, Chang WC, Fang YF, Chen CT, Lang JY, et al. Epidermal growth factor receptor potentiates MCM7-mediated DNA replication through tyrosine phosphorylation of Lyn kinase in human cancers. Cancer Cell 2013, 23:796-810. 


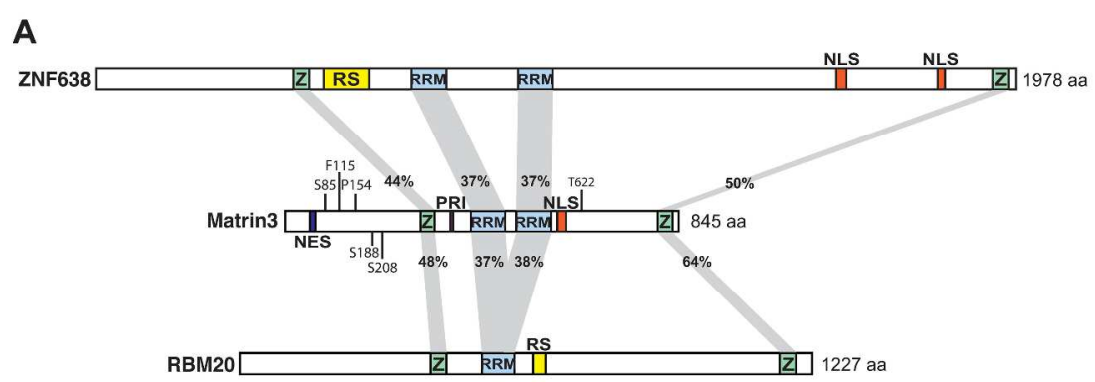

B

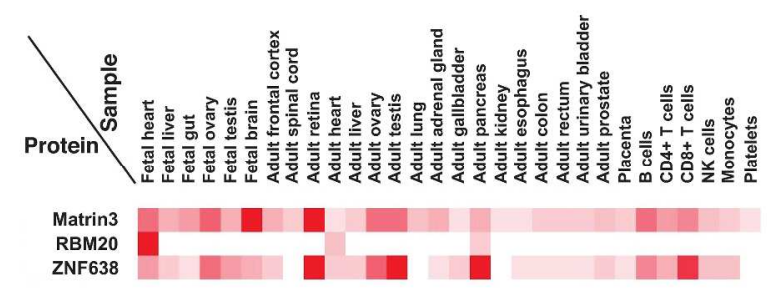

Figure 1 - Matrin 3 and related protein structure and expression $297 \times 420 \mathrm{~mm}$ ( $300 \times 300$ DPI) 
A

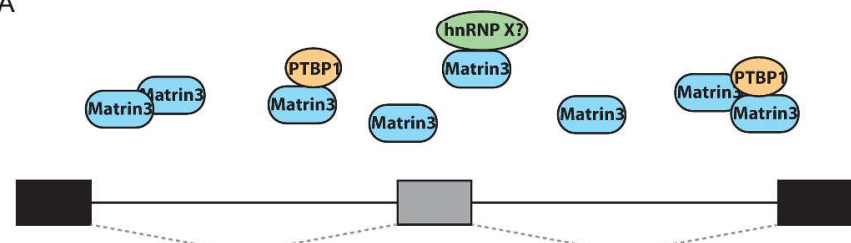

B

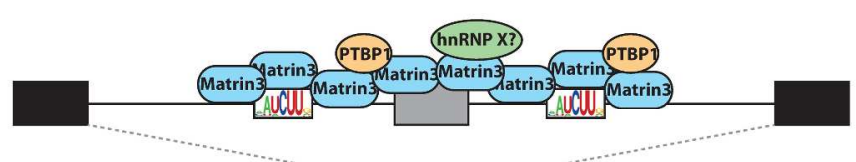

Figure 2 - Splicing Regulation by Matrin3 297x420mm (300 x 300 DPI) 